\title{
NDRProfile Schema Version 1.0 User Guide
}

\author{
Betty Harvey \\ Joshua Lubell \\ Puja Goyal \\ KC Morris
}




\section{NDRProfile Schema Version 1.0 User Guide}

Betty Harvey Electronic Commerce Connection, Inc.

Email: harvey@eccnet.com

Joshua Lubell Puja Goyal KC Morris Manufacturing Systems Integration Division National Institute of Standards and Technology Email: \{lubell,pgoyal,kcm\}@nist.gov

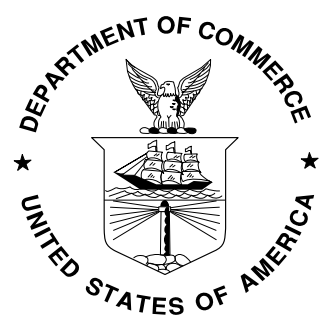

U.S. Department of Commerce Carlos M. Gutierrez, Secretary 


\title{
NDRProfile Schema Version 1.0 User Guide
}

\author{
Betty Harvey <harvey@eccnet . com> \\ Joshua Lubell <lubell@nist.gov> \\ Puja Goyal <pgoyal@nist.gov> \\ KC Morris $<k c m e n i s t . g o v>$
}

\begin{abstract}
The NDRProfile schema provides a common syntax for exchanging, managing, and reusing XML Schema naming and design rules (NDRs). NDRProfile, used by NIST's Quality of Design (QOD) application as a format for import and export of rule sets, is also useful for general exchange of NDRs and, with accompanying stylesheets and other materials, provides an environment for authoring and formatting NDR documentation. This paper describes the content of the NDRProfile schema and its various uses.
\end{abstract}

\section{Table of Contents}

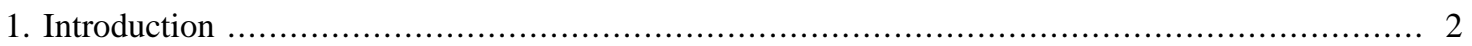

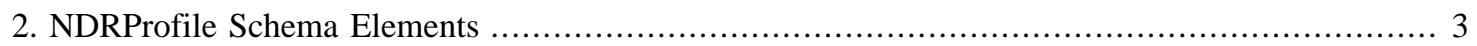

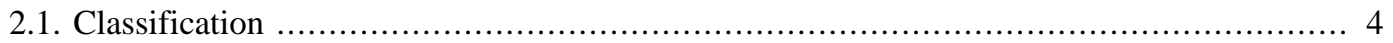

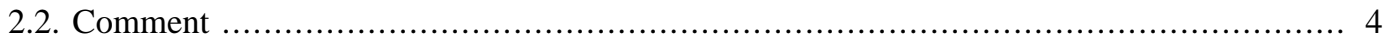

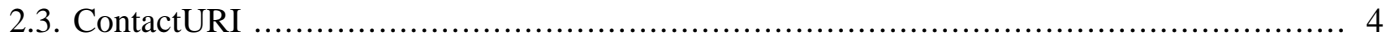

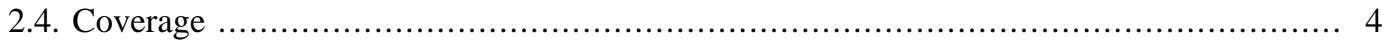

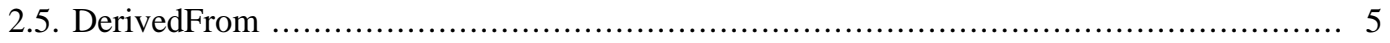

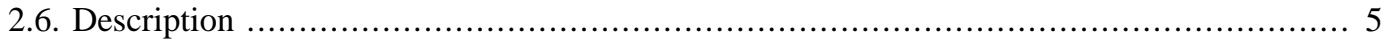

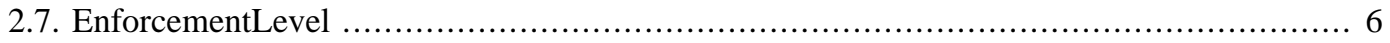

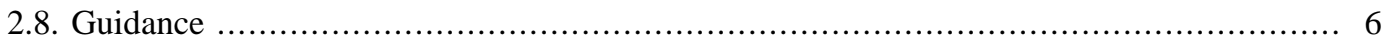

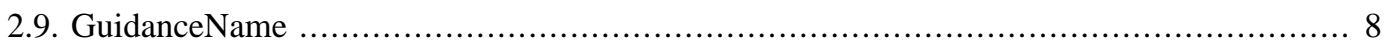

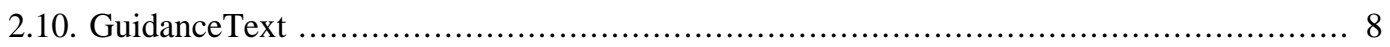

2.11. Justification

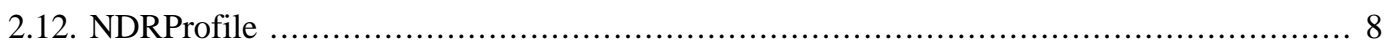

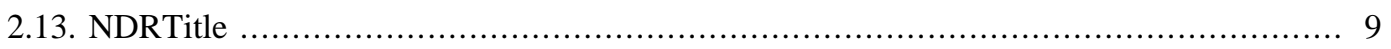

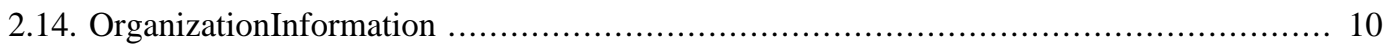

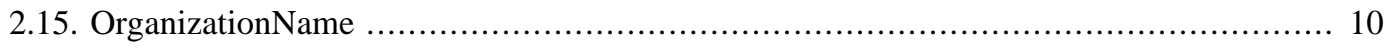

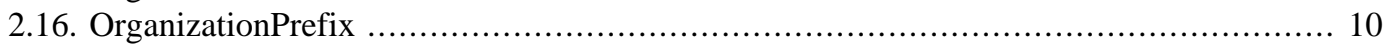

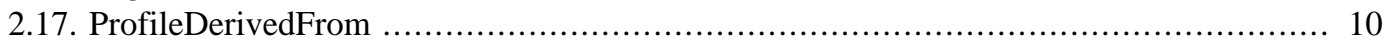

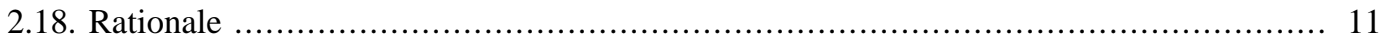

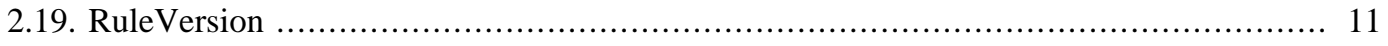

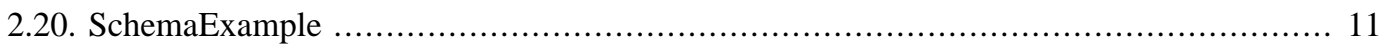

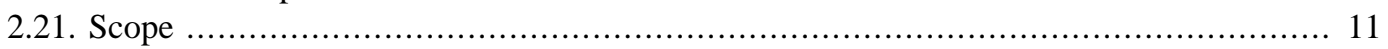

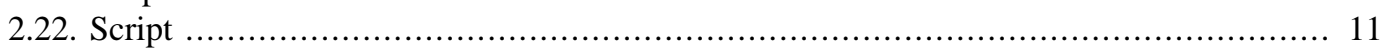

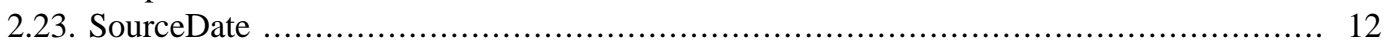

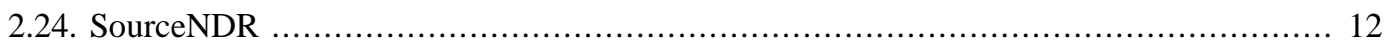

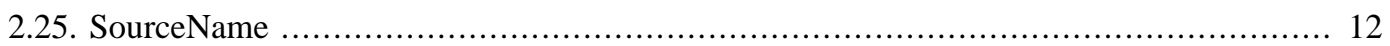




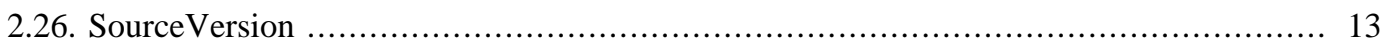

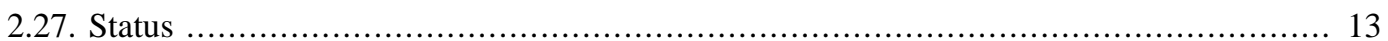

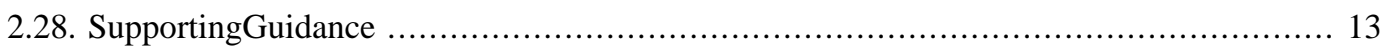

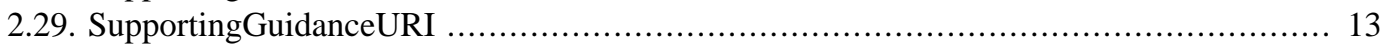

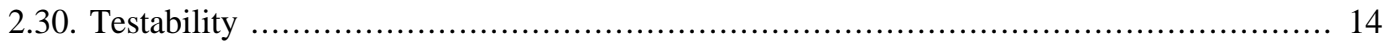

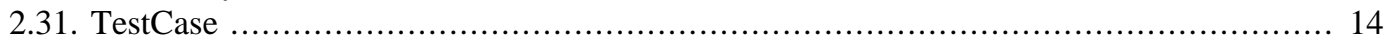

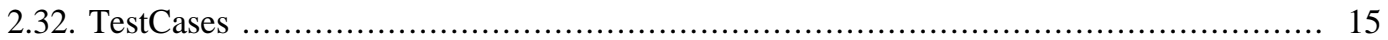

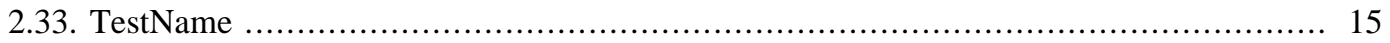

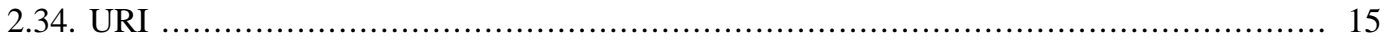

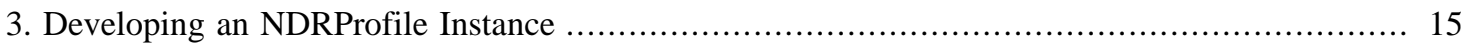

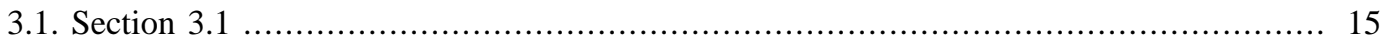

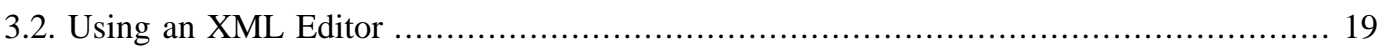

3.3. Spreadsheet Transformation ............................................................ 19

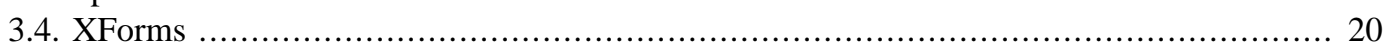

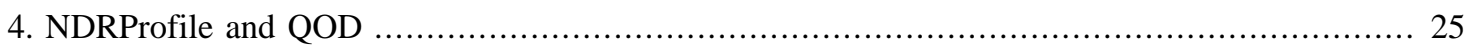

4.1. QOD Handling of Imported Guidance and Test Cases ..................................... 25

4.2. NDRProfile Identifiers ............................................................... 26

5. Lessons Learned and the Future of NDRProfile ............................................ 26

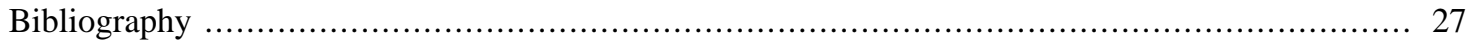

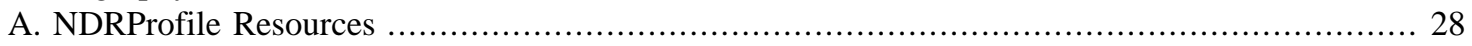

\section{Disclaimer}

Mention of commercial products or services in this document does not imply approval or endorsement by NIST, nor does it imply that such products or services are necessarily the best available for the purpose.

\section{Introduction}

XML schema [1] Naming and Design Rules (NDRs) [2] are intended to help organizations enforce best practices, a particular modeling methodology, or conformance to common nomenclature policies. But there are barriers that can prevent NDRs from improving schema interoperability and quality:

- NDR proliferation - no single rule set meets everyone's requirements.

- Absence of NDR document structure - NDR documentation typically is created using a word processor and exists only in a proprietary binary format.

- Lack of automated rule enforcement - the onus is on XML schema developers to ensure that their schemas obey (sometimes ambiguously worded) NDR rules.

- Limited versioning and traceability - it is cumbersome to compare NDRs with one another or to explicitly specify rule reuse.

NIST created the Quality of Design (QOD) web application (http://www.nist.gov/qod) to address these barriers. QOD provides a mechanism for checking XML schema design quality against NDRs in a collaborative environment. Plans for the next version include better support for sharing and publication of rules with the intention of maintaining an extensive set of reusable test cases.

Recognizing that some groups might want to develop their own XML schema design quality testing tools rather than use QOD, NIST subsequently created NDRProfile - an XML schema providing a common format in which NDRs can be exchanged, managed and reused. NDRProfile provides the following capabilities: 
- Import and export of rule collections into and from the QOD.

- The ability to publish NDR documentation from XML.

- The ability to exchange NDR rule collections, including tests for the rules.

The NDRProfile schema's target audience includes NDR authors, groups wishing to exchange, share, or reuse rule sets, and users of schema quality assurance tools such as QOD that import/export XML conforming to the NDRProfile. Although the NDRProfile schema's development was inspired by QOD, the schema can be used independently of QOD, either as a means of authoring an NDR document or as a vehicle for sharing rules between NDR Profile schema-compliant software applications.

This document is intended both for NDR authors as well as for developers of NDR authoring or testing software. The document contains the following sections:

- Section 2, "NDRProfile Schema Elements" provides information about the elements and attributes that are available in the NDRProfile schema.

- Section 3, "Developing an NDRProfile Instance" describes the various methods for developing an NDRProfile document.

- Section 4, "NDRProfile and QOD" describes QOD and discusses using NDRProfile for import and export. Non-QOD users may wish to skip reading this section.

- Section 5, "Lessons Learned and the Future of NDRProfile" concludes the user guide with some thoughts on improving NDRProfile.

The NDRProfile schema and the accompanying stylesheets and resources mentioned in Section 3, "Developing an NDRProfile Instance" are available in a single package available for download from http:// qod.sourceforge.net.

\section{NDRProfile Schema Elements}

This section provides an alphabetical listing of all elements and attributes included in the NDRProfile XML Schema. Each element is defined in its own subsection. The information provided for each element includes:

\section{Element name}

2. Brief definition of each element

3. Data type if the element has simple content (character data with no sub-elements)

4. A graphical representation of the content if the element contains sub-elements

5. A table of attributes providing information about each attribute, if the element has attributes

6. A list of parent elements.

The table below shows how to interpret the symbols used in content diagrams. 


\begin{tabular}{|l|l|}
\hline Symbol & Meaning \\
\hline Guidance & $\begin{array}{l}\text { A rectangle represents an element whose name is the string inside the } \\
\text { rectangle. A number range underneath the box specifies the number } \\
\text { of occurrences (zero or more for Guidance in the example on the } \\
\text { left). If no range is given, the element occurs exactly once. }\end{array}$ \\
\hline $\begin{array}{l}\text { An octagon containing a horizontal line connecting a series of dots } \\
\text { represents an XML Schema sequence element. }\end{array}$ \\
\hline
\end{tabular}

The best way for a user to get an overview of the schema is to first read the definition for NDRProfile, the root element of an NDRProfile document instance. Another good element to look at for gaining an overall sense of the schema is Guidance.

\subsection{Classification}

Definition: Classification specifies a category for the guidance rule. The taxonomy of categories is determined by the NDR developers.

Data type: $x s d$ :string

\section{Parent Elements}

- Guidance

\subsection{Comment}

Definition: Comment is used to specify any information about a guidance rule not specifiable using other NDRProfile elements.

Data type: xsd:string

\section{Parent Elements}

- Guidance

\subsection{ContactURI}

Definition: ContactURI is used to provide a web or email address for an organizational point of contact.

Data type: $x s d: a n y U R I$

\section{Parent Elements}

- OrganizationInformation

\subsection{Coverage}

Definition: Coverage defines what portion of the rule the test case covers. 
Data type: $x s d: s t r i n g$

\section{Parent Elements}

- TestCase

\subsection{DerivedFrom}

Definition: DerivedFrom contains information about the original source of the rule when this is a new rule that is a modification of a rule from a different NDR. This element is used for traceability.

\section{Content:}

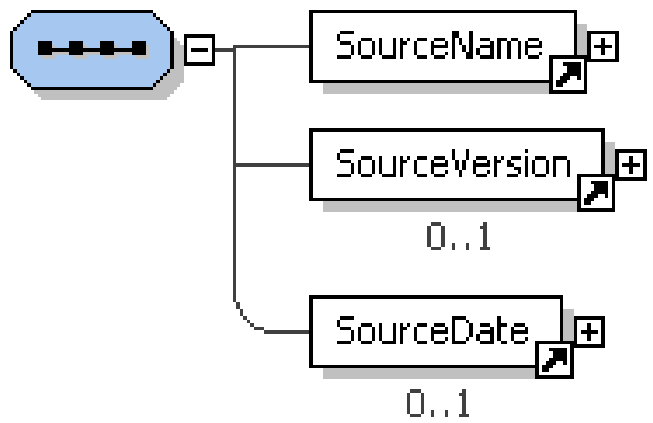

\begin{tabular}{|c|c|c|c|}
\hline Attribute Name & Definition & Allowed Values & Default Value \\
\hline $\begin{array}{l}\text { QODUniqueIdentifier } \\
\text { (optional) }\end{array}$ & $\begin{array}{l}\text { QODUniqueIdentifier is an } \\
\text { optional attribute containing a unique } \\
\text { identifier assigned by the QOD } \\
\text { web application. This attribute is } \\
\text { populated by QOD when the rule } \\
\text { is exported from QOD into a valid } \\
\text { NDRProfile instance. }\end{array}$ & xsd:string & None \\
\hline URI & $\begin{array}{l}\text { URI is an XML unique ID type. The } \\
\text { first character of an XML ID must be } \\
\text { a letter followed by any XML name } \\
\text { characters (letter, number, ., -, _, or:) }\end{array}$ & xsd:anyURI & None \\
\hline
\end{tabular}

\section{Parent Elements}

- Guidance

\subsection{Description}

Definition: Description specifies text documenting the NDR.

Data type: xsd:string

\section{Parent Elements}

- NDRProfile 
- TestCase

\subsection{EnforcementLevel}

Definition: EnforcementLevel defines whether the guidance rule is mandatory, optional, or recommended.

Data type: Mandatory, Optional, or Highly_Recommended

\section{Parent Elements}

- Guidance

\subsection{Guidance}

Definition: Guidance is the top level element for an NDR rule. It represents a test requirement.

\section{Content:}




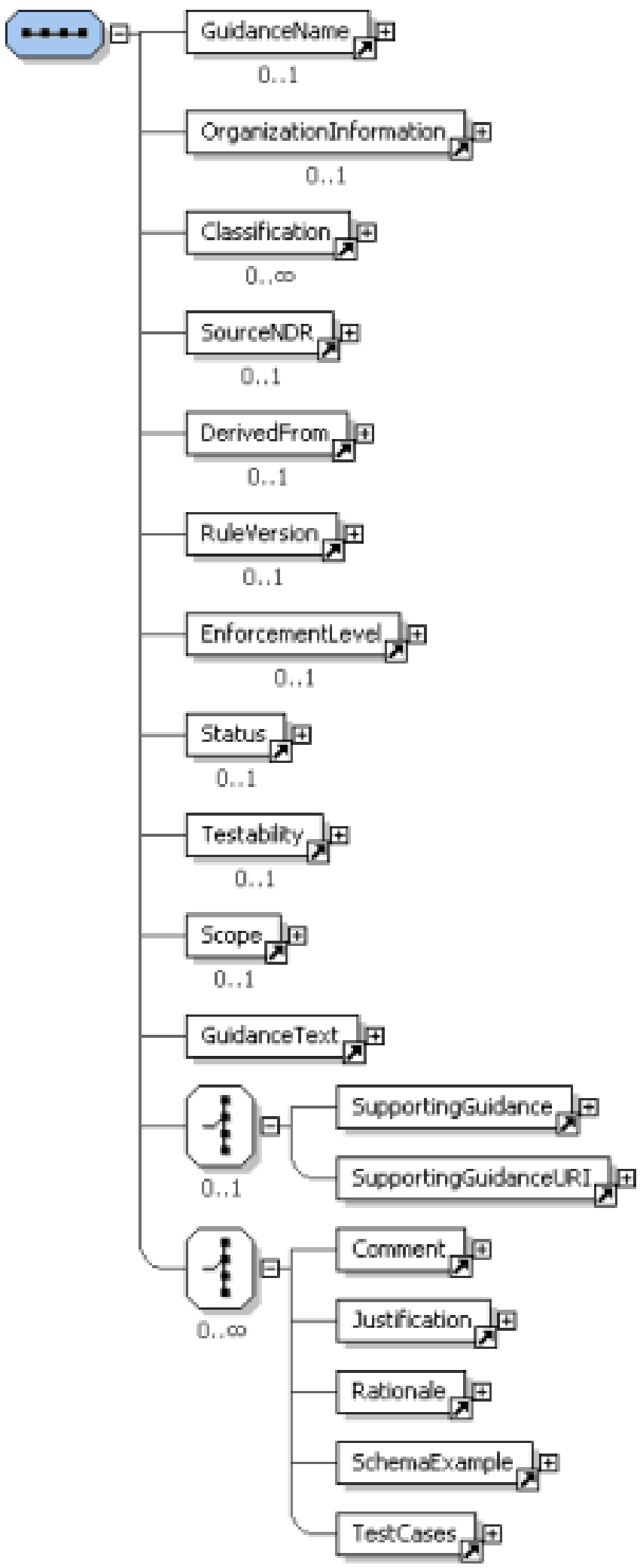




\begin{tabular}{|l|l|l|l|}
\hline Attribute Name & Definition & Allowed Values & Default Value \\
\hline documentationID & $\begin{array}{l}\text { documentationID is a link to } \\
\text { optional) }\end{array}$ & $\begin{array}{l}\text { NDsd:string } \\
\text { the "NDRDecumentation. The use of } \\
\text { the "documentationID" attributes } \\
\text { provides the mechanism to link } \\
\text { internally to the NDR documentation } \\
\text { from the NDRProfile schema. }\end{array}$ & None \\
\hline guidanceID & $\begin{array}{l}\text { guidanceID is a link to a unique } \\
\text { identifier within the NDRProfile } \\
\text { document and should be unique for } \\
\text { each rule or guidance. }\end{array}$ & xsd:ID & None \\
\hline
\end{tabular}

${ }^{\text {a }}$ A guidanceID must conform to additional contraints if the NDRProfile is to be imported into NIST's QOD web application.

\section{Parent Elements}

- NDRProfile

\subsection{GuidanceName}

Definition: GuidanceName defines the succinct title for the rule.

Data type: xsd:string

\section{Parent Elements}

- Guidance

\subsection{GuidanceText}

Definition: GuidanceText is an English language specification of the rule.

Data type: xsd:string

\section{Parent Elements}

- Guidance

\subsection{Justification}

Definition: Justification provides additional supporting information, beyond the value for Rationale, regarding the reason for the guidance.

Data type: xsd:string

\section{Parent Elements}

- Guidance

\subsection{NDRProfile}

Definition: NDRP rofile is the root element for a document conforming to the NDRProfile schema. 


\section{Content:}

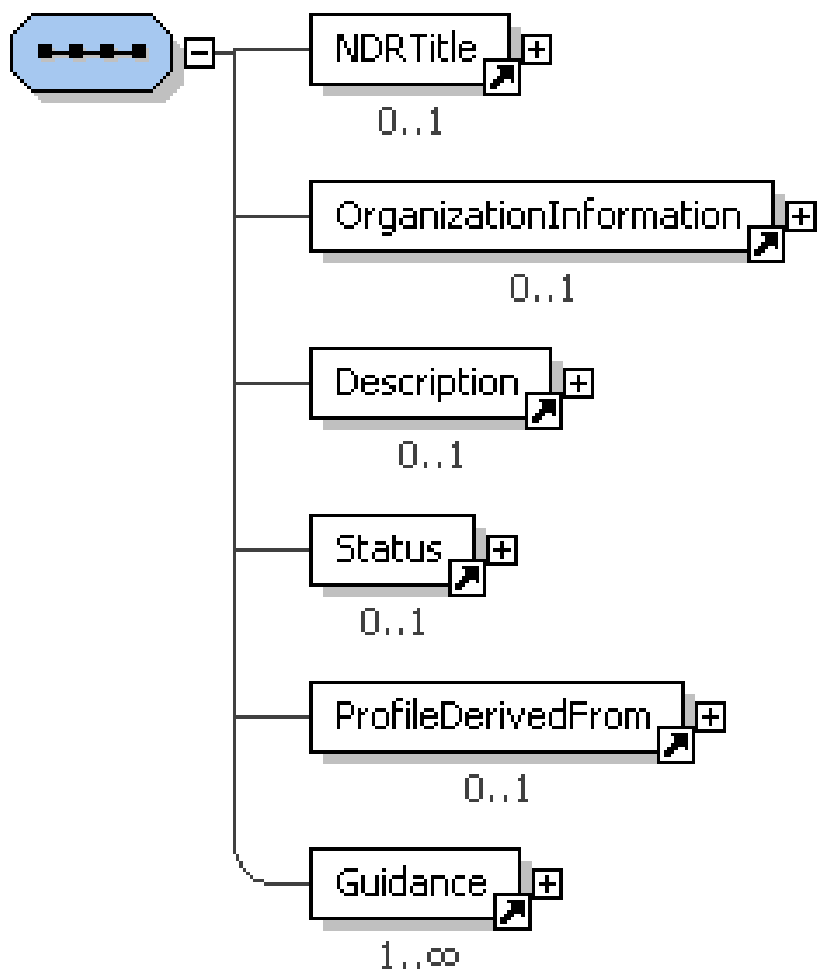

\begin{tabular}{|l|l|l|l|}
\hline Attribute Name & Definition & Allowed Values & Default Value \\
\hline NDRProfileID & $\begin{array}{l}\text { NDRProfileID is a unique } \\
\text { identifier for the NDR. }\end{array}$ & Xsd:ID \\
\hline $\begin{array}{l}\text { NDRProfileBaseURI } \\
\text { (optional) }\end{array}$ & $\begin{array}{l}\text { NDRProfileBaseURI contains } \\
\text { the location of the NDRProfile. }\end{array}$ & xsd:anyURI \\
\hline $\begin{array}{l}\text { NDRDocumentBaseURI } \\
\text { (optional) }\end{array}$ & $\begin{array}{l}\text { NDRDocumentBaseURI points to } \\
\text { the location of the documentation for } \\
\text { the NDR. It is envisioned that the } \\
\text { documentation will be encoded using } \\
\text { DocBook. }\end{array}$ & None \\
\hline
\end{tabular}

\section{Parent Elements}

- Root Element

\subsection{NDRTitle}

Definition: NDRTitle specifies a brief description of the rule set. If the NDRProfile represents a formal NDR document, NDRTitle should contain the document's official title.

Data type: xsd:string

\section{Parent Elements}

- NDRProfile 


\subsection{OrganizationInformation}

Definition: OrganizationInformation specifies data about the entity responsible for the rule set or guidance rule.

\section{Content:}

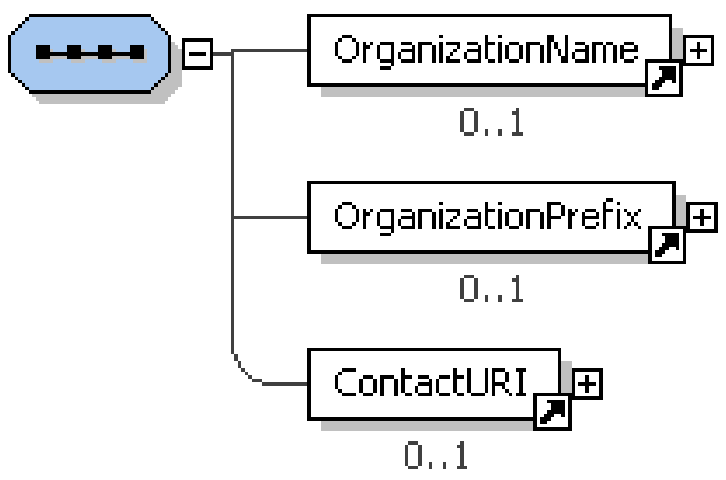

\section{Parent Elements}

- NDRProfile

- Guidance

\subsection{OrganizationName}

Definition: OrganizationName defines the name for an organization.

Data type: $x s d: s t r i n g$

\section{Parent Elements}

- OrganizationInformation

\subsection{OrganizationPrefix}

Definition: OrganizationPrefix defines a character sequence for an organization. This character sequence is used internally by QOD to create identifiers for guidance created within QOD by the organization.

Data type: xsd:string

\section{Parent Elements}

- OrganizationInformation

\subsection{ProfileDerivedFrom}

Definition: ProfileDerivedFrom specifies the original source of the NDR.

Content:

\begin{tabular}{|l|l|l|l|}
\hline Attribute Name & Definition & Allowed Values & Default Value \\
\hline URI & URI is a valid URI. & xsd:anyURI & None \\
\hline
\end{tabular}




\section{Parent Elements}

- NDRProfile

\subsection{Rationale}

Definition: Rationale provides a reason for creating the rule. Although Rationale's data type permits any string value, our intention for the next version of NDRProfile is to restrict the value to an enumerated set similar to that proposed in [3].

Data type: xsd:string

\section{Parent Elements}

- Guidance

\subsection{RuleVersion}

Definition: RuleVersion defines the version number of the guidance rule.

Data type: xsd:integer

\section{Parent Elements}

- Guidance

\subsection{SchemaExample}

Definition: SchemaExample associates a sample schema with a guidance rule in order to give an example of rule compliance or violation.

Data type: xsd:string with white space preserved

\section{Parent Elements}

- Guidance

\subsection{Scope}

Definition: Scope defines the level of granularity of the guidance, i.e., whether it applies to a single value or field, an aggregate component (such as a complex type definition), or to the XML schema as a whole.

Data type: $x s d$ :string

\section{Parent Elements}

- Guidance

\subsection{Script}

Definition: Script specifies code implementing a test case. The code should be written in the language specified by the TestCase element's ruleType attribute.

Data type: xsd:string with white space preserved 


\section{Parent Elements}

- TestCase

\subsection{SourceDate}

Definition: SourceDate is the date the derived rule was published.

Data type: xsd:date

\section{Parent Elements}

- DerivedFrom

- SourceNDR

\subsection{SourceNDR}

Definition: SourceNDR specifies the original source of the rule. It is used to indicate if the rule is a copy of a rule already existing in another NDR.

\section{Content:}

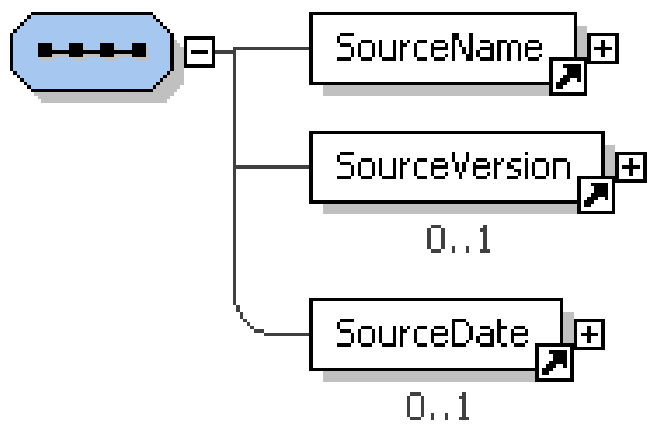

\begin{tabular}{|l|l|l|l|}
\hline Attribute Name & Definition & Allowed Values & Default Value \\
\hline QODUniqueIdentifier & $\begin{array}{l}\text { QODUniqueIdentifier is an } \\
\text { optional attribute which contains the } \\
\text { unique identifier assigned by QOD. } \\
\text { This attribute will be populated by } \\
\text { QOD when the rule is exported } \\
\text { from QOD into a valid NDRProfile } \\
\text { instance. }\end{array}$ & None \\
\hline URI & $\begin{array}{l}\text { URI is an XML unique ID type. The } \\
\text { first character of an XML ID must be } \\
\text { a letter followed by any XML name } \\
\text { characters (letter, number,.,-,_, or:: }:\end{array}$ & xsd:anyURI & None \\
\hline
\end{tabular}

\section{Parent Elements}

- Guidance

\subsection{SourceName}

Definition: SourceName is the name of the NDR the rule was derived from. 
Data type: $x s d: s t r i n g$

\section{Parent Elements}

- DerivedFrom

- SourceNDR

\subsection{SourceVersion}

Definition: SourceVersion is the version of the NDR the rule was derived from.

Data type: xsd:string

\section{Parent Elements}

- DerivedFrom

- SourceNDR

\subsection{Status}

Definition: Status specifies the standing of a guidance rule or NDRProfile, e.g., "Draft."

Data type: xsd:string

Parent Elements

- NDRProfile

- Guidance

\subsection{SupportingGuidance}

Definition: SupportingGuidance allows the association of additional information with a guidance rule.

Data type: xsd:string

\section{Parent Elements}

- Guidance

\subsection{SupportingGuidanceURI}

Definition: SupportingGuidanceURI allows a URI to be provided for additional documentation for the guidance.

Data type: xsd:anyURI

\section{Parent Elements}

- Guidance 


\subsection{Testability}

Definition: Testability defines whether a rule can be programmatically evaluated. Actual assessment or confirmation of a rule's quality is outside the scope of NDRProfile.

Data type: Fully-Testable, Partially-Testable, or Untestable

\section{Parent Elements}

- Guidance

\subsection{TestCase}

Definition: TestCase is a computer executable script, i.e., a binding of the guidance to a specific implementation. A test case determines whether an XML schema complies with or violates the guidance rule.

\section{Content:}

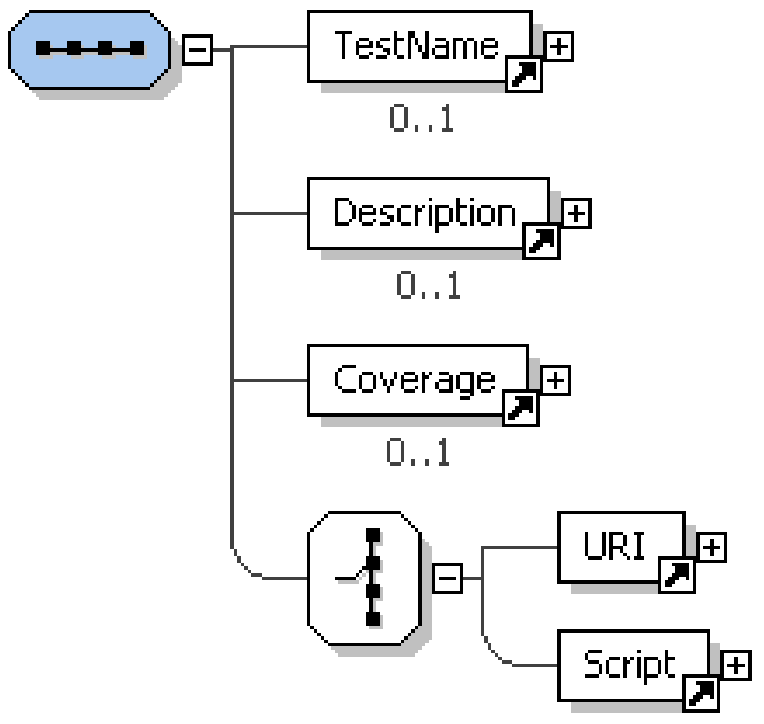

\begin{tabular}{|l|l|l|l|}
\hline Attribute Name & Definition & Allowed Values & Default Value \\
\hline ruleType & $\begin{array}{l}\text { ruleType identifies the type of } \\
\text { validation rule that has been created. } \\
\text { If "Other" is selected, the attribute } \\
\text { 'typeOther' should be used to identify } \\
\text { the type of validation rule that was } \\
\text { created. }\end{array}$ & $\begin{array}{l}\text { Schematron1.5, } \\
\text { Jess, Other }\end{array}$ & None \\
\hline typeOther & $\begin{array}{l}\text { typeOther identifies a validation } \\
\text { rule that has been created in another } \\
\text { language besides Schematron [4] [5] } \\
\text { and Jess [6]. }\end{array}$ & xsd:string & None \\
\hline testCaseID & $\begin{array}{l}\text { The testCaseID is a unique identifier } \\
\text { for the test case. It must conform to } \\
\text { the XML ID rule construct. }\end{array}$ & xsd:ID & None \\
\hline
\end{tabular}




\section{Parent Elements}

- TestCases

\subsection{TestCases}

Definition: TestCases defines one or more test cases.

\section{Content:}

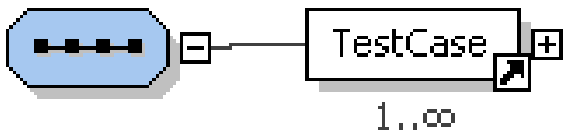

\section{Parent Elements}

- Guidance

\subsection{TestName}

Definition: TestName defines a name that a user can assign to a test.

Data type: xsd:string

\section{Parent Elements}

- TestCase

\subsection{URI}

Definition: URI defines a link to a single test case.

Data type: xsd:anyURI

\section{Parent Elements}

- TestCase

\section{Developing an NDRProfile Instance}

This section describes various ways users can develop a document conforming to the NDRProfile schema document. The conforming document can then be formatted for viewing using the NDRProfile-toHTML.XSI stylesheet, imported into QOD (see Section 4, "NDRProfile and QOD"), or imported into some other NDRProfile-aware application.

NDRProfile-to-HTML.xsl and other files mentioned in this section are listed in Appendix A, NDRProfile Resources, and are included in the NDRProfile download package on SourceForge.

\subsection{DocBook Documentation}

Organizations can develop their NDR using the standard DocBook schema. DocBook [7] is a standard for developing technical documentation. We have developed an XML Style Language Tranformation (XSLT) [8] stylesheet that extracts the rules from a DocBook instance and produces XML conforming to the NDRProfile schema. 
Several standards bodies, such as the Univeral Business Language Technical Committee [http:// www.oasis-open.org/committees/ubl], have started developing and maintaining their NDRs using the DocBook standard. The use of DocBook XML can provide a mechanism for automatically extracting an NDRProfile document from the full NDR documentation.

We have developed an XSLT stylesheet (NDRDocumentDocbook-to-NDRProfile.xsl) to convert a DocBook tagged NDR to the NDRProfile XML document. You should be able to use any XSLT processor to create an NDRProfile xml file from a DocBook XML document, provided the DocBook is tagged as described below.

The figure below shows how rules are extracted from the DocBook XML document. The resulting output from the XSLT extraction is a fully conformant NDRProfile XML document.

\section{Figure 1. DocBook Documentation to NDRProfile}

\section{DocBook.xsd}

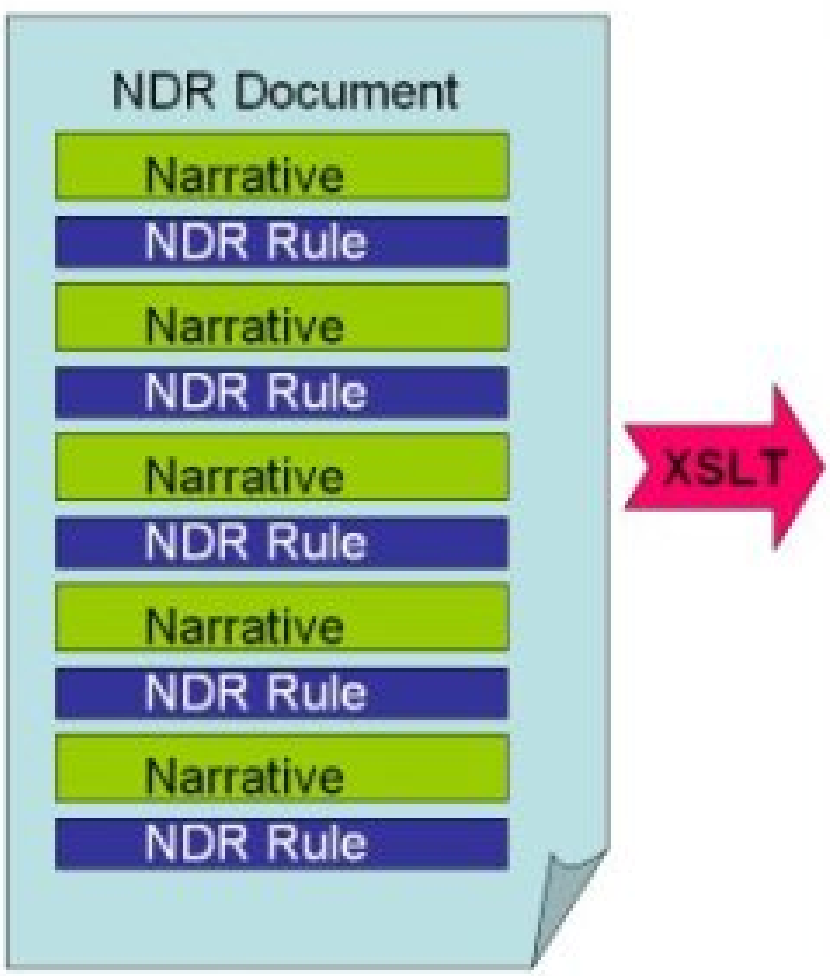

NDRProfile.xsd

NDR Profile

NDR Rule

NDR Rule

NDR Rule

NDR Rule

NDR Rule

For this method to be used, rules and rule prefixes must be identified within the document instance using DocBook's role attribute. The XSLT stylesheet uses the role values to transform the DocBook XML into NDRProfile guidance rules.

The XSLT extraction uses the blockquote element with a role attribute value of Rule to extract the rule. The blockquote element must also include a unique identifier for uniquely identifying rules.

\section{Note}

Both the role and id attributes are optional attributes within the DocBook schema. Therefore, you must ensure that all rules include the role and id attributes. We have developed a 
Schematron [4] schema, CheckDocbookNDRRules.sch, that can be used to ensure that the blockquote element includes the required information to support conversion to an NDRProfile.

\section{Example 1. Using blockquote with role and id attributes to define a rule}

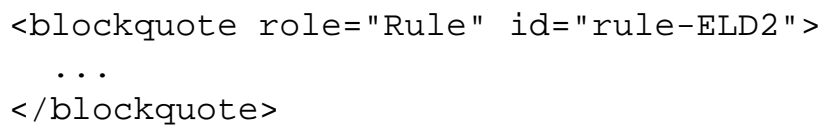

DocBook uses the para element to define a paragraph. The text documenting the rule should be included in a para element.

If your NDR standard uses rule prefixes to disambiguate rules within your NDR, the emphas is element should be used with the role attribute set to rule-prefix within the para element. In the following example, the NDR uses "ELD2" as a unique identifier with implied semantics. "ELD" is an abbreviation for "Element Declaration." "2" is appended to the identifier to differentiate the rule from other rules in the NDR dealing with element declaration.

\section{Example 2. Using para to provide text of a rule}

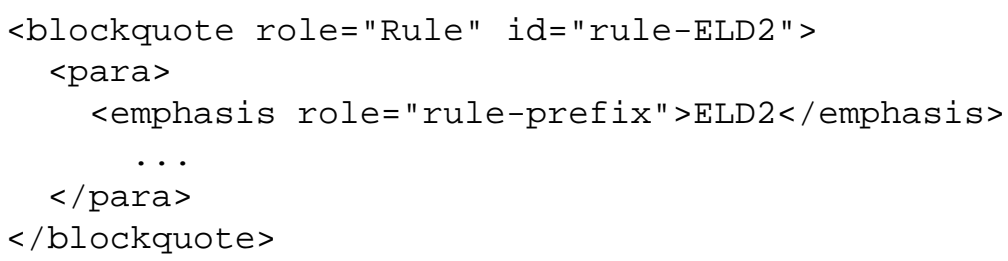

Schematron test cases can be included in the documentation using the DocBook element programlisting. The programlisting element should be included after the para element. The example below shows the complete DocBook markup of an NDR rule that includes a Schematron test case. A CDATA section is used to prevent the Schematron from being parsed during transformation from DocBook to NDRProfile. CDATA sections specify to an XML processor that characters such as angle brackets and ampersands should not be treated as markup. [9] 


\section{Example 3. Rule with Schematron test case}

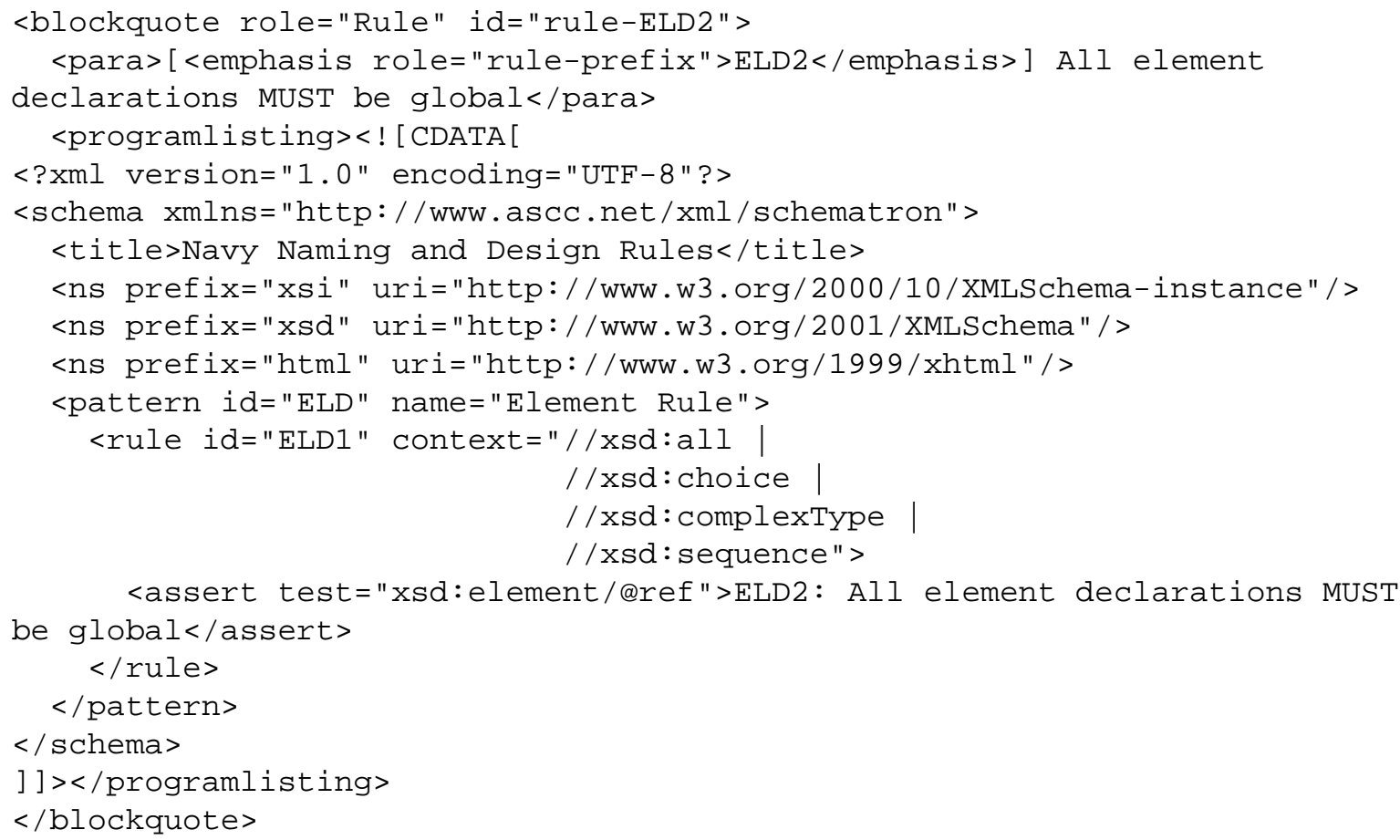

Your favorite XSLT processor can be used to create the NDRProfile XML document from the NDR DocBook document. Below is a command line example (with a newline added for readability) using Saxon [10] (an XSLT processor written in Java [11]), of converting an NDR document created with DocBook into an NDRProfile document. For guidance using other XSLT processors, refer to their documentation.

java -jar saxon.jar -o NDRProfile.xml NDRDocument.xml

NDRDocumentDocbook-to-NDRProfile.xsl

Saxon interprets the command as follows:

\begin{tabular}{|l|l|}
\hline Command Part & Explanation \\
\hline java - jar saxon.jar & $\begin{array}{l}\text { Uses the Java Runtime Environment to invoke the Saxon XSLT } \\
\text { processor }\end{array}$ \\
\hline -o NDRProfile.xml & $\begin{array}{l}\text { Sends the result tree to an output file called NDRProfile.xml. The } \\
\text { name of the file is arbitrary and user controlled }\end{array}$ \\
\hline NDRDOcument.xml & The input file - a complete NDR document tagged using DocBook \\
\hline $\begin{array}{l}\text { NDRDocumentDocbook-to- } \\
\text { NDRProfile.xsl }\end{array}$ & The XSLT stylesheet performing the conversion \\
\hline
\end{tabular}

The blockquote element will be transformed into the following NDRProfile output:

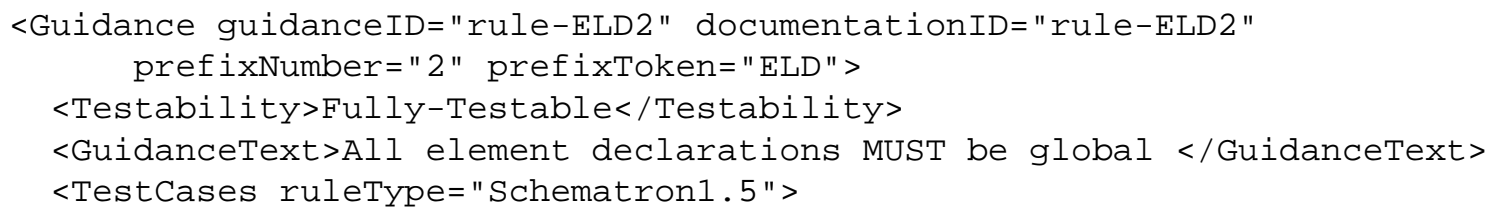




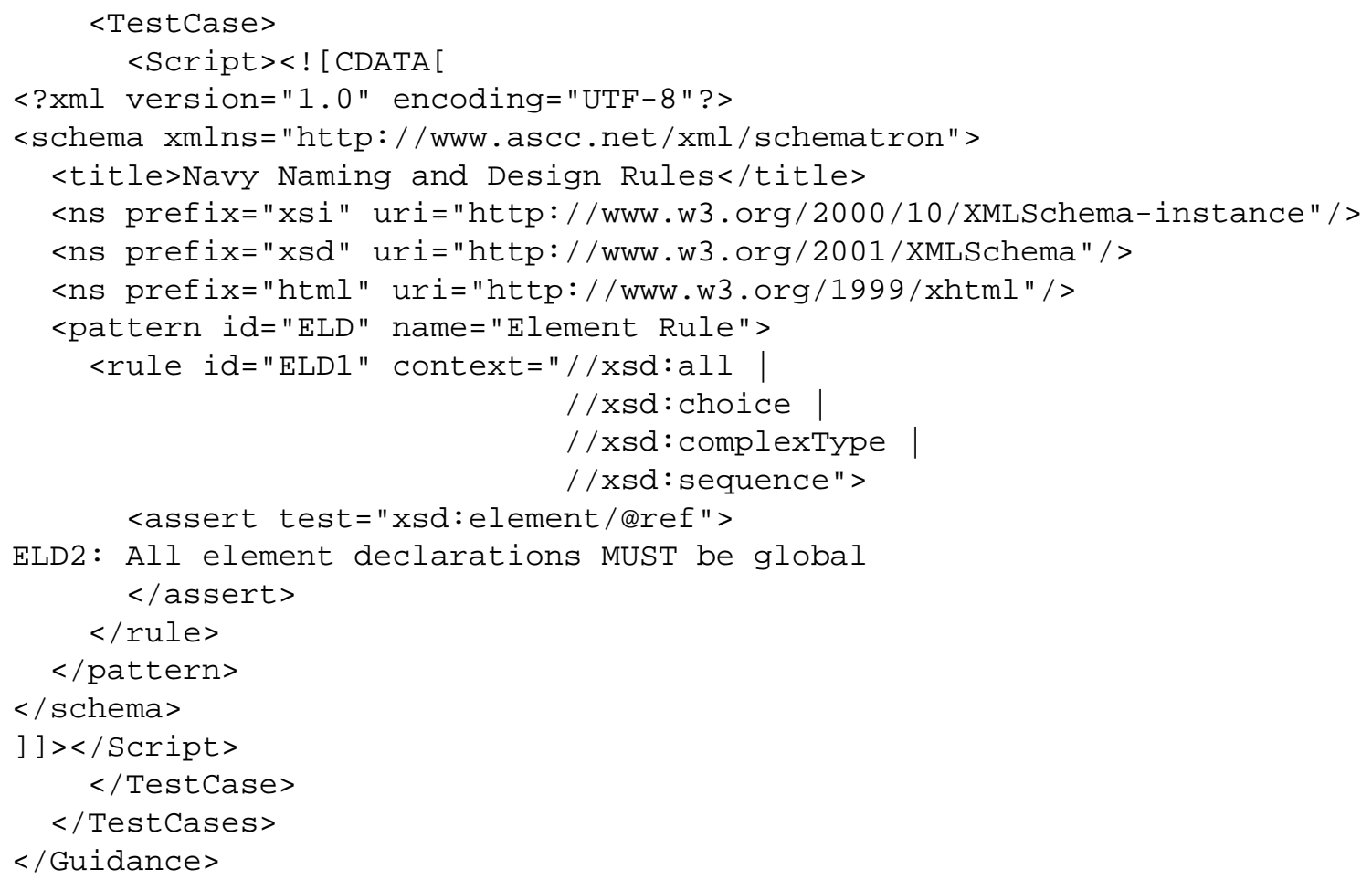

\subsection{Using an XML Editor}

Any XML editor can be used to develop an NDRProfile document. We have developed a cascading stylesheet (CSS) [12] and XSLT stylesheet to format an NDRProfile document for viewing in a browser or editor. These stylesheets should work with any XML editor supporting XSLT or CSS.

\subsection{Spreadsheet Transformation}

Several standards organizations use spreadsheets to maintain NDR rules. We have created a spreadsheet template in Microsoft's Excel format [13] based on review of the several spreadsheets currently being used. The spreadsheet will allow users to create an NDR using the Excel spreadsheet application. We have also developed an XSLT stylesheet that will transform the NDR spreadsheet to NDRProfile XML. This XSLT can be used to transform a generic NDR to the NDRProfile document instance as long as the fields in the spreadsheet are the same as our fields. If an organization decides to modify the spreadsheet, they can modify the XSLT to support their new structure.

The following table shows the correspondence between spreadsheet columns and NDRProfile schema objects.

\begin{tabular}{|l|l|l|}
\hline Column & Title & NDRProfile Mapping \\
\hline A & Guidance ID & Guidance/@guidanceID \\
\hline B & Guidance Name & Guidance/GuidanceName \\
\hline C & Derived From & Guidance/DerivedFrom \\
\hline D & Enforcement & Guidance/EnforcementLevel \\
\hline E & Guidance Text & Guidance/GuidanceText \\
\hline F & Testability & Guidance/Testablity \\
\hline G & Comment & Comment \\
\hline
\end{tabular}


The spreadsheet must be saved as an XML file prior to conversion. You can save the spreadsheet to an XML file in Excel by:

1. Clicking on File -> Save As.

2. Type the name of the file in the "File name" block.

3. Change the "Save as type" to XML Data.

\section{Click Save.}

Once the spreadsheet has been saved to an XML file, it is converted to the Excel XML Format. The XSLT stylesheet, Excel-to-NDRProfile.xsl, converts the XML from the Excel format to the NDRProfile XML format. The conversion can be accomplished using an XSLT processor. The following command uses Saxon (newline added for readability):

java -jar saxon.jar -o NDRProfile.xml NDRProfile-Template.xml

Excel-to-NDRProfile

The command is interpreted as follows:

\begin{tabular}{|l|l|}
\hline Command Part & Explanation \\
\hline java - jar saxon.jar & Invoke Saxon \\
\hline -o NDRProfile.xml & Send the result tree to the output file NDRP rofile.xml. \\
\hline NDRProfile-Template.xml & The input file - in this case, a spreadsheet exported to XML \\
\hline Excel-to-NDRProfile.xsl & The XSLT stylesheet which performs the conversion \\
\hline
\end{tabular}

\subsection{XForms}

An XForms [14] form has been developed for creating an NDRProfile using a web browser. The form requires a browser such as Mozilla Firefox with client-side support for the XForms standards.

\section{Note}

To use this form, Mozilla's XForms plugin [15] must be installed. Information about obtaining the plugin can be found at http://www.mozilla.org/projects/xforms. Follow the instructions for installing the plug-in. The form document, NDRProfile-Form. xhtml, uses a template file NDRProfile.xml for the initial population of the fields.

The XForm is designed in 2 pages $^{1}$. The first page, shown below, obtains metadata concerning the entire NDR. The second page obtains individual Guidance elements, i.e., NDR rules.

\footnotetext{
${ }^{1}$ This form is intended as a proof of concept. Therefore, we have not paid much attention to user interface design principles or readability. Also, as of November 2008, the Mozilla XForms extension is still pre-version 1.0. We present screen shots of the form and show the generated XML in order to illustrate the potential of XForms as a low-cost easy-to-use authoring solution.
} 
Figure 2. Page 1 of Mozilla Form

\section{Developing An NDRProfile}

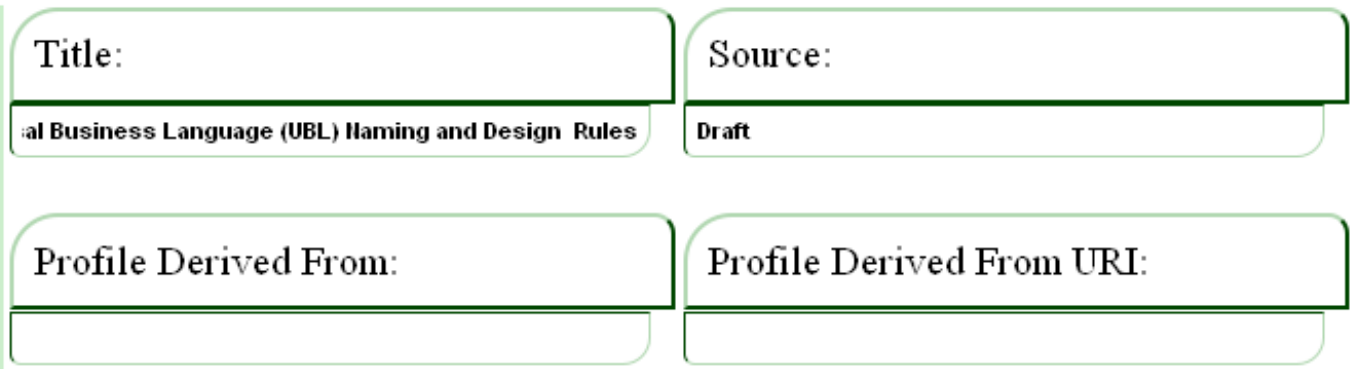

\begin{tabular}{|l|l|}
\hline Profile Date: & Documentation Location: \\
\hline $2006-09-08$ & nttp://www.oasis-open.org/committees/download.php \\
\hline
\end{tabular}

\begin{tabular}{|l|l|}
\hline NDR URI: & NDR Identifier: \\
\hline Imittees/download.php/10323/cd-UBL-IIDR- 1.0Rev1c.pdf & IIDR100 \\
\hline
\end{tabular}

Contact: Description:

mavis.Cournane@cognitran.com

istruction of XML components for the UBL vocabulary.

NDR Status:

Draft|

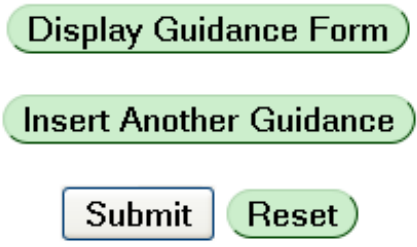

The second page of the form allows creation of the components for the Guidance portions of the NDR. Page 2 is a repeating group that will allow the user to add as many Guidance elements as needed. 
Figure 3. Page 2 (First Screen Shot) Mozilla Form

\section{Guidance}
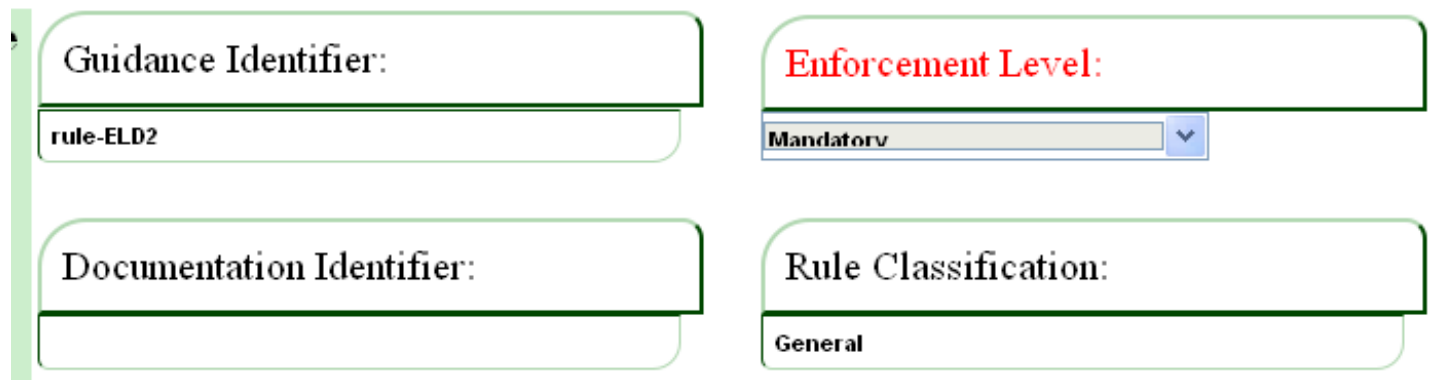

Rule Classification:

NDR Prefix Token:

NDR Prefix Number:

ELD

1

Rationale for Rule:

Comments: 


\section{Figure 4. Page 2 (Second Screen Shot) Mozilla Form}

\begin{tabular}{|l|}
\hline Rule Status: \\
Draft \\
Gu idance Text : \\
\hline All element declarations must be global \\
\hline
\end{tabular}

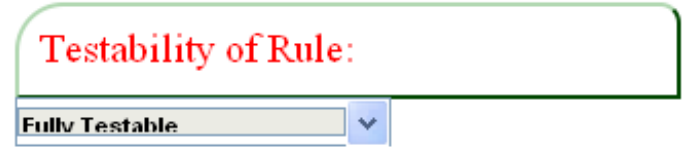

Schematron Test Case:

\begin{tabular}{|c|c|}
\hline $\begin{array}{l}\text { //xsd:complexType | } \\
\text { //xsd:sequence"> } \\
\text { <assert test="usd:element/@ref"> } \\
\text { ELD2: All element declarations MUST be } \\
\text { global</assert> } \\
</ \text { rule> } \\
<\text { /pattern> } \\
\text { </schema> }\end{array}$ & $\hat{\imath}$ \\
\hline
\end{tabular}

Scope of Rule:

Complete this section if rule was derived from another rule.

Derived Profile Name:

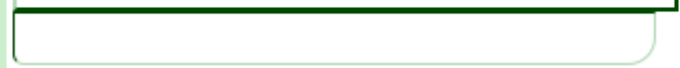

Derived Profile Date:

\section{Derived Profile Version:}

\section{Display Guidance Form ) \\ Insert Another Guidance \\ Submit Reset}

When the user clicks the "Submit" button, an NDRProfile XML document is created in a file called NDRProfile-new.xml. If you want to modify the resulting NDRProfile XML document, copy this file to NDRProfile.xml in the directory where NDRProfile-Form. xhtml file is located. 
Below is the XML file generated from the screenshots shown above:

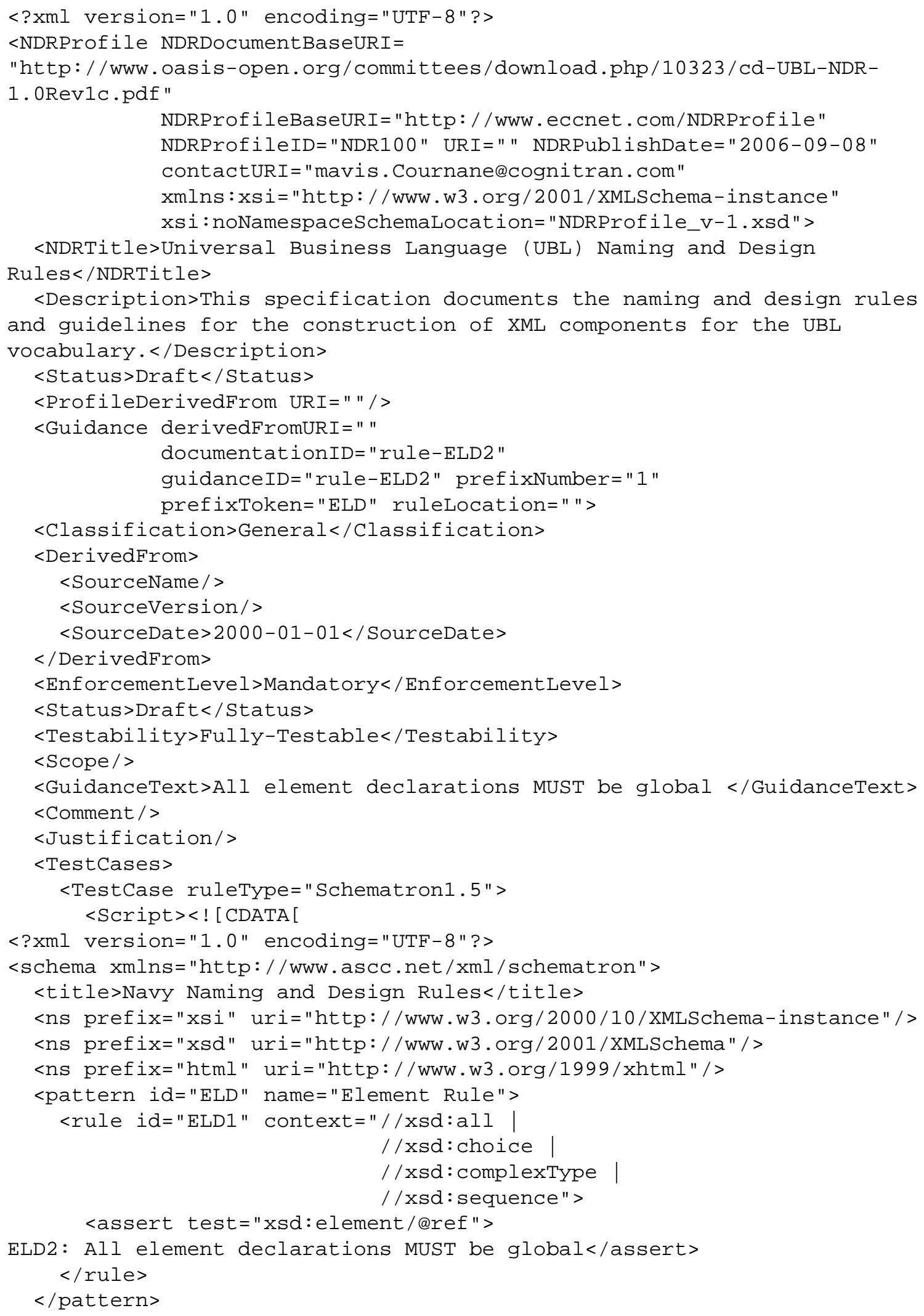




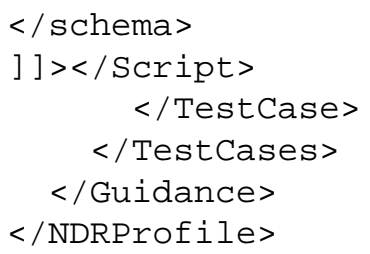

\section{NDRProfile and QOD}

QOD assists standards developers in consistently using XML Schema for the specification of information. Consistent design of XML schemas within an organization or single integration project can reduce the number and the severity of interoperability problems. In addition, this consistency makes the XML schema easier to extend, understand, implement, and maintain; and, it paves the way for automated testing and mapping. Applying best XML schema design practices and following agreed-upon naming conventions are ways to achieve this design consistency.

Users can develop an NDRProfile using QOD. QOD enables a user to create Test Cases and Guidance Rules (called "test requirements" in the QOD web application), associate them with each other, and store them in a central repository. Test cases can then be selected and grouped into an NDRProfile (or "test profile" in QOD terminology). This QOD test profile can then be exported to an XML file conforming to NDRProfile. QOD can import NDRProfile instances as well.

\subsection{QOD Handling of Imported Guidance and Test Cases}

QOD's import functionality allows a user to upload an NDRProfile written in XML and conforming to the NDRProfile schema. This enables making changes to an NDRProfile offline and importing these changes back into QOD for testing and validation. If a user uploads a new NDRProfile (one which does not currently exist as a test profile in the QOD repository), a new test profile is created. Based on whether the rules or test cases already exist in the QOD database, different options are displayed to the user to determine how these elements are handled. These options are described below.

Add The NDRProfile element does not already exist in QOD. If the user clicks "Add," the element is added to the database, and the user is assigned ownership of it.

Insert The NDRProfile element already exists in QOD, but is not associated with the NDRProfile being uploaded. Selecting the insert option establishes an association in QOD between the existing item and the uploaded NDRProfile.

Update The NDRProfile element already exists in QOD, is owned by the user, and is already associated with the NDRProfile being uploaded. Selecting this option updates QOD to match the NDRProfile element. If the user is not the owner of the NDRProfile element, the update option is not available.

Remove This option appears when both of these conditions occur:

- The user uploads an NDRProfile whose identifier matches that of an existing QOD test profile (s)he already owns.

- The QOD test profile is associated with guidance rules or test cases not included in the uploaded NDRProfile.

The option appears for each item associated with the QOD test profile but not in the uploaded NDRProfile. Clicking "Remove" removes the connection between the guidance rule or test case from the test profile. 


\section{Note}

This will not remove the rule or test case completely from the QOD database.

\subsection{NDRProfile Identifiers}

The existence of items in the QOD database is determined by the ID assigned to the item, either as the NDRProfileID, the guidanceID, or the testCaseID. To ensure QOD consistency when creating the NDRProfile outside of the QOD environment, the user is free to assign any ID to the NDRProfileID, while the guidanceID and testCaseID must be a concatenation of the Guidance's organizationPrefix and SourceVersion elements, and the prefixToken and prefixNumber attributes from the NDRProfile. However, when creating test requirements and test cases within the QOD, these IDs are automatically structured and generated based on user input. The composition of the guidanceID and testCaseID are as follows:

guidanceID: organizationPrefix-SourceVersion-[prefixToken]-prefixNumber

Where:

- organizationPrefix is the prefix related to the name of the organization that created the NDR

- SourceVersion is the NDR version

- prefixToken (optional) and prefixNumber create the unique identifier for a rule in the NDR.

Example of a guidanceID: OAGI-9.0-R-16

testCaseID: guidanceID_implementationNumber

Where:

- ImplementationNumber is a number that should be unique among test cases implementing the same test requirement. For example, if test case $\mathrm{A}$ and test case $\mathrm{B}$ both implement test requirement $\mathrm{C}$, suggested implementation numbers for test case A and test case B would be 1 and 2 respectively. Note ImplementationNumber is not an element in the NDRProfile.

Example of a testCaseID: OAGI-9.0-R-16-1

\section{Lessons Learned and the Future of NDRProfile}

QOD informed NDRProfile's design, and our implementation experience provided important and beneficial insight toward specifying the schema. On the other hand, the NDRProfile schema may suffer from too much QOD influence in its identifier constructs. For example, the OrganizationPrefix element and QODUniqueIdentifier attribute both assume QOD-specific implementation details that might not be relevant for other NDRProfile use cases. We hope that others will develop XML schema design quality tools supporting NDRProfile. Only through multiple independent implementations can we determine whether the current NDRProfile identifier representations are helpful for other tools besides QOD.

NDRProfile's DerivedFrom element provides a rudimentary way to say that one guidance rule is a modification of another rule. It would be useful if NDRProfile could represent additional information about the modification. For example, two rules may be identical in all respects except that they refer to different 
namespaces. Or one rule may be the same as another rule, but with an additional restriction added. We are currently studying NDRs from multiple sources with the ultimate goal of incorporating a taxonomy of reuse scenarios into the NDRProfile schema to better support management of rule sets.

The focus of this document has been on the content of the NDRProfile schema, use of the schema for creating new NDRProfile instances, and NDRProfile's relationship with the QOD application. In Section 1, "Introduction" we enumerated four barriers to NDR effectiveness: proliferation of rule sets, lack of NDR document structure, lack of automation, and poor versioning and traceability. The current NDRProfile schema addresses the first three barriers by specifying an XML language for representing sets of guidance rules and associating guidance rules with computer-interpretable code to determine compliance. Our vision is for NDRProfile to continue to evolve through results from further analysis of NDRs and of inter-rule relationships, and from additional implementation experience.

\section{Acknowledgment}

The authors are grateful to Mark Palmer for his thorough and helpful review of an earlier draft of this document.

\section{Bibliography}

[1] World Wide Web Consortium. XML Schema Part 0: Primer Second Edition. W3C Recommendation 28 October 2004, http://www.w3.org/TR/xmlschema-0/ .

[2] Cover Pages. Naming and Design Rules. http://xml.coverpages.org/ndr.html .

[3] B. Kulvatunyou. K.C. Morris. XML Schema Design Quality Test Requirements. National Institute of Standards and Technology. NISTIR 7175. October 2004. http://www.mel.nist.gov/msidlibrary/publications.html .

[4] ISO/IEC 19757-3. Information technology - Document Schema Definition Languages (DSDL) - Part 3: Rule-based validation - Schematron. First edition, 2006-06-01, http://standards.iso.org/ittf/PubliclyAvailableStandards .

[5] Rick Jelliffe. The Schematron Assertion Language 1.6. http://xml.ascc.net/schematron/ .

[6] Jess, the Rule Engine for the Java Platform. http://herzberg.ca.sandia.gov/ .

[7] Norman Walsh and Leonard Muellner. DocBook: The Definitive Guide. O'Reilly \& Associates. Updated: Wed, 11 Oct 2006, Version 2.0.16, http://www.docbook.org/tdg .

[8] World Wide Web Consortium. XSL Transformations (XSLT) Version 1.0. W3C Recommendation 16 November 1999, http://www.w3.org/TR/xmlschema-0/ .

[9] World Wide Web Consortium. Extensible Markup Language (XML) 1.0 (Fourth Edition). W3C Recommendation 16 August 2006, edited in place 29 September 2006, http://www.w3.org/TR/xml .

[10] The SAXON XSLT and XQuery Processor. http://saxon.sourceforge.net .

[11] Wikipedia. Java Virtual Machine. http://en.wikipedia.org/wiki/Java_Virtual_Machine .

[12] World Wide Web Consortium. Cascading Style Sheets Level 2 Revision 1 (CSS 2.1) Specification. W3C Candidate Recommendation 17 July 2007, http://www.w3.org/TR/CSS21 .

[13] Wikipedia. Microsoft Excel. http://en.wikipedia.org/wiki/Microsoft_Excel .

[14] World Wide Web Consortium. XForms 1.0 (Third Edition). W3C Recommendation 29 October 2007, http:// www.w3.org/TR/xforms . 
[15] Mozilla.org. Mozilla XForms Project. http://www.mozilla.org/projects/xforms/ .

\section{A. NDRProfile Resources}

This appendix lists the stylesheets and templates available for working with the NDRProfile schema. The resources were discussed in Section 3, "Developing an NDRProfile Instance" and are included with the NDRProfile Schema package, available from the QOD Sourceforge website (http://qod.sourceforge.net/).

NDRProfile-to-HTML.xsl

NDRProfile_v-1.cSs

NDRDocumentDocbook-to-

NDRProfile.xsl

CheckDocbookNDRRules.sch

NDRProfile-Template.xls

Excel-to-NDRProfile.xsl

NDRProfile-Form.xhtml

NDRProfile.xml
XSLT stylesheet that can be used to either display an NDRProfile instance within a browser supporting XSLT or to convert the NDRProfile instance to HTML.

CSS stylesheet for formatting an NDRProfile document for viewing in an XML editor or browser.

XSLT stylesheet to convert an NDR DocBook instance to an instance of NDRProfile. The DocBook instance must use role and id attributes as explained in Section 3.1.

Schematron schema for ensuring that NDR documentation marked up using Docbook contains attributes needed for conversion to NDRProfile.

Excel template for authoring an NDRProfile document.

XSLT stylesheet for converting an NDRProfile spreadsheet exported as XML into a document conforming to the NDRProfile schema.

XForms form for creating an NDRProfile instance.

Template for initial population of the fields of NDRPRofileForm.xhtml. 